\title{
Broadband Dipole-Loop Combined Nanoantenna Fed by Two-Wire Optical Transmission Line
}

\author{
Janilson L. de Souza, ${ }^{1,2}$ Karlo Q. da Costa, ${ }^{1}$ Victor Dmitriev, ${ }^{1}$ and Felipe Bamberg ${ }^{1}$ \\ ${ }^{1}$ Department of Electrical Engineering, Federal University of Para, Belém, PA, Brazil \\ ${ }^{2}$ Department of Electrotechnology, Federal Institute of Education, Science and Technology of Para, Tucuruí, PA, Brazil \\ Correspondence should be addressed to Janilson L. de Souza; janilson@ufpa.br
}

Received 21 September 2016; Revised 31 December 2016; Accepted 9 January 2017; Published 28 February 2017

Academic Editor: Yuan Yao

Copyright (C) 2017 Janilson L. de Souza et al. This is an open access article distributed under the Creative Commons Attribution License, which permits unrestricted use, distribution, and reproduction in any medium, provided the original work is properly cited.

\begin{abstract}
This paper presents a broadband nanoantenna fed by a two-wire optical transmission line (OTL). The antenna is defined by a combination of a dipole and a loop, where only the dipole element is connected to the OTL. The analysis is fulfilled by the linear method of moments with equivalent surface impedance to model the conductors. Firstly, the nanoantenna alone is investigated, where the input impedance, current distribution, reflection coefficient, fractional bandwidth, radiation efficiency, and radiation pattern are analyzed. Then, the input impedance matching of this antenna with the OTL is considered. In this case the current, near field distribution, radiation pattern, and reflection coefficient are calculated for different geometrical parameters. The results show that the loop inserted in the circuit can increase the bandwidth up to $42 \%$ and decreases the reflection coefficient in the OTL to $-25 \mathrm{~dB}$.
\end{abstract}

\section{Introduction}

Recently, accompanying the development of plasmonic technology, the study of antennas exceeded the microwave barrier, reaching the infrared and optical regions. In these regions, there is a profusion of possibilities and proposals of applications. These antennas are devices designed to transmit, receive, and manipulate optical fields in nanometric scale going beyond the diffraction limit $[1,2]$. For example, using optical antennas with broadband spectral response one can develop more efficient photovoltaic devices [3,4] and amplify fluorescent emission spectroscopy [5] and Raman scattering (SERS) [6]. For these and other applications, several models of optical broadband antennas can be used as, for example, in [7] where a broadband monopole nanoantenna is proposed. The increase in bandwidth for the antenna is achieved by varying the antenna dimensions. In [8], a trapezoidal plasmonic nanoantenna is presented, where the enlarged band is achieved by overlapping different dipole resonances. In [9], a large bandwidth is obtained in the plasmonic nanoantenna with six and eight particles with a common gap. In [10], a nanoantenna formed by an array of equally spaced nanorods of variable length is presented. In this case, the increase in bandwidth is achieved by the nanoantenna arrangement.

Optical antennas can also be used in conjunction with plasmonic waveguides for designing highly integrated photonic signal processing systems, because plasmonic waveguides can efficiently handle optical fields in nanometric scale beyond the diffraction limit as well [11]. For example, a plasmonic waveguide in the form of a two-wire optical transmission line (OTL) is used in papers [12-14] to make the interconnection with optical antennas forming an optical nanocircuit. Here, the optical antennas work as terminal elements transforming the far field radiation into guided waves and vice versa. In the above works, the impedance matching between the plasmonic waveguide and the optical antenna was analyzed. The optical circuit comprises one nanoantenna for reception and another one for emission connected to a two-wire OTL. In [12], the impedance matching was attained varying the geometric dimensions of the nanodipole and the gap of the OTL for a fixed frequency. In [13] some conclusions are presented about the conditions where a better impedance matching between the emitter dipole and the OTL for a fixed frequency can be obtained. 
Besides, an analysis of the excitation is fulfilled as well. In [14], the nanocircuit is fed by an aperture probe, where the coupling between the aperture probe and the receiving antenna is modeled by an equivalent voltage source. In this article, the authors analyzed the impedance matching varying the geometrical dimensions of the nanodipole for a given frequency range. In the aforementioned works, the optical circuits and optical antennas with broadband spectral response were not analyzed.

In this work, a theoretical analysis of a broadband nanoantenna in an optical nanocircuit is presented. The broadband nanoantenna is formed by a combination of a loop and a dipole antenna. It is connected to a two-wire optical transmission line. The nanoantenna is obtained by placing the electric nanodipole in the center of the rectangular loop with a power source connected to the nanodipole. This geometry was used due to its simplicity in manufacturing and calculus as compared, for example, with the geometries of $[7,8]$. Notice that this type of antennas for microwave region was analyzed in [15].

The numerical analysis is performed via a simple and efficient computational method based on linear method of moments (MoM) [16]. The presented results concern the nanoantenna bandwidth and quantitative analysis of the impedance matching of the OTL with the nanoantenna. Some results are compared with the simulations by the commercial software Comsol [17]. The results show that the loop used in the circuit increases the bandwidth of the nanoantenna to $42 \%$ and decreases the voltage reflection coefficient of the optical nanocircuit up to $-25 \mathrm{~dB}$.

\section{Theoretical Development}

This section presents the geometry of the problem and the model of the plasmonic nanocircuit by the MoM. In this model, the Lorentz-Drude model is used to represent the complex permittivity of the metal, which is used in the calculation of the surface impedance of the cylindrical conductors of the circuit. The linear MoM is used to solve the $1 \mathrm{D}$ integral equation of the electric field with linear approximation of the longitudinal current, sinusoidal basis functions, and test functions of rectangular pulse [16].

2.1. Description of the Problem. The nanocircuit structure is shown in Figure 1, where cylindrical gold conductors located in free space form the structure. In this figure, a voltage source $V_{s}$ applied in the gap $d$ feeds the nanocircuit. The nanocircuit is composed of a two-wire OTL connected to a broadband nanoantenna formed by the combination of a dipole antenna (straight dipole) and a loop antenna (rectangular loop). The voltage source is centered at the origin of the reference system, the OTL and the dipole are on the plane $z=0$, and the loop is located in the plane $z=d_{e}$; that is, $d_{e}$ is the distance between the dipole and the loop.

2.2. Method of Moments Model. For conducting the analysis of the nanocircuit shown in Figure 1, the linear method of moments is applied to solve the $1 \mathrm{D}$ integral equation of the electric field with linear approximation of the longitudinal

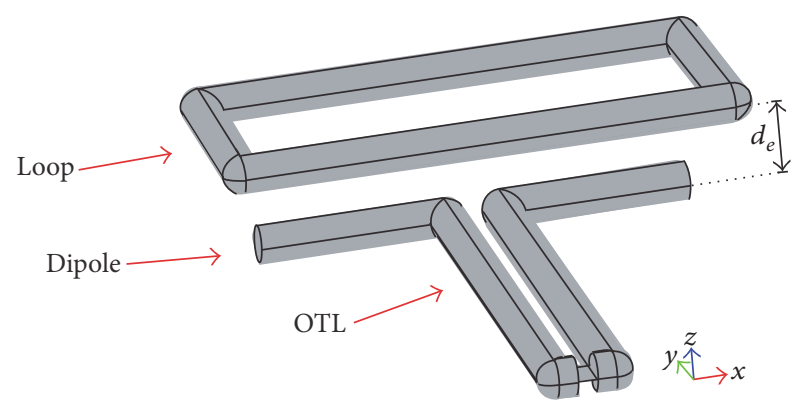

FIGURE 1: Nanocircuit, consisting of a two-wire optical transmission line (OTL), a dipole antenna (straight dipole), and a loop antenna (rectangular).

current, sinusoidal basis functions, and test functions of the rectangular pulse [16]. Figure 2 shows the geometry as seen from above in the direction of the $z$-axis of the nanocircuit. The nanocircuit dimensions are as follows: $L$ and $a_{L}$ are the length and the radius of the OTL, respectively; $h=h_{d}+a_{L}$ and $a_{d}$ are the length of one arm and radius of the electrical nanodipole, respectively; $d_{L}$ is the distance between the axes of the OTL; $D=d_{L}-2 a_{L}$ is the distance between the surfaces of the OTL; $d=d_{L} / 2$ is the gap of the voltage source; $W_{e}+2 a_{e}$, $H_{e}+2 a_{e}$, and $a_{e}$ are the width, length, and radius of the loop, respectively; $d_{W}$ and $d_{H}$ are the distances as seen from above the structure between the surfaces of the dipole and the loop. The side view of the nanoantenna without the OTL is shown in Figure 3.

Gold is the material chosen to make the cylindrical nanostructures of the circuit considered in this work. It is represented by the complex permittivity model of LorentzDrude $\varepsilon_{1}=\varepsilon_{0} \varepsilon_{r 1}$ [18]:

$$
\varepsilon_{r 1}=\varepsilon_{\infty}-\frac{\omega_{p 1}^{2}}{\omega^{2}-j \Gamma_{D} \omega}+\frac{\omega_{p 2}^{2}}{\omega_{0}^{2}-\omega^{2}+j \gamma_{L} \omega},
$$

where the constants are $\omega_{0}=2 \pi c / \lambda_{0}$, where $\lambda_{0}=450 \mathrm{~nm}$ and $c=3 \times 10^{8} \mathrm{~m} / \mathrm{s}, \varepsilon_{\infty}=8, \omega_{p 1}=13.8 \times 10^{15} \mathrm{~s}^{-1}, \omega_{p 2}=$ $45 \times 10^{14} \mathrm{~s}^{-1}, \Gamma_{D}=1.075 \times 10^{14} \mathrm{~s}^{-1}$, and $\gamma_{L}=9 \times 10^{14} \mathrm{~s}^{-1}$.

We use the following boundary condition for the electric field on the surface of the linear conductor:

$$
\left(\bar{E}_{s}+\bar{E}_{i}\right) \cdot \bar{a}_{l}=Z_{s} I,
$$

where $\bar{a}_{l}$ is the unit vector tangent to the conductor surface, $\bar{E}_{i}$ is the external incident electric field, $\bar{E}_{s}$ is the scattered electric field, $I$ is the longitudinal electric current, and $Z_{s}$ is the surface impedance describing the losses in the metal. This impedance $Z_{s}$ is determined approximately by considering the conductor as a cylindrical waveguide in the $\mathrm{TM}_{01}$ mode [19], which is the main mode of the problem for frequency range $100-400 \mathrm{THz}$, disregarding other modes that have no significant contribution. In this case the impedance is given by

$$
\begin{aligned}
& Z_{s}=\frac{T J_{0}(T a)}{2 \pi a j \omega \varepsilon_{1} J_{1}(T a)}, \\
& T=k_{0} \sqrt{\varepsilon_{r 1}}, k_{0}=\omega \sqrt{\mu_{0} \varepsilon_{0}}, \quad \varepsilon_{1}=\varepsilon_{0} \varepsilon_{r 1},
\end{aligned}
$$




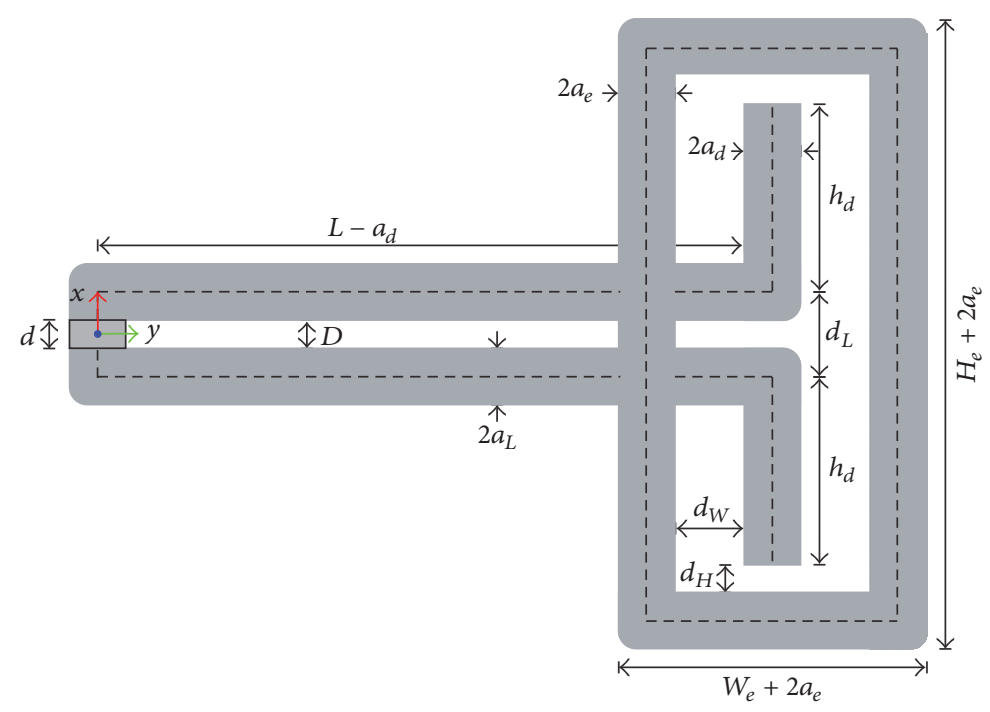

FIgURE 2: Top view of the cylindrical plasmonic nanocircuit.

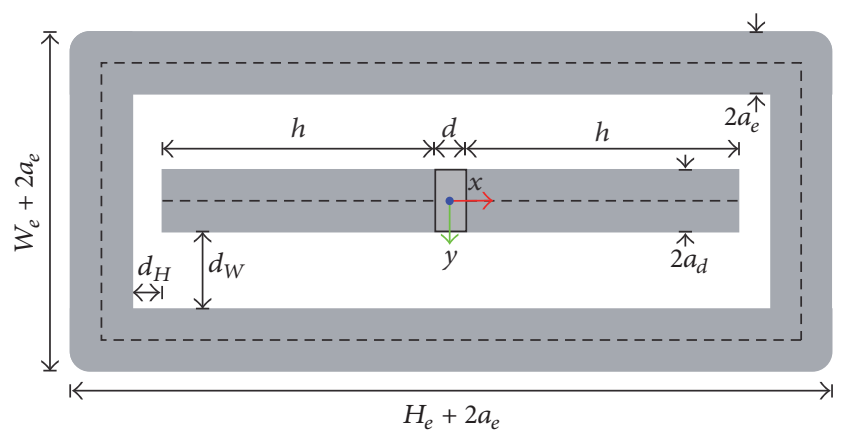

FIGURE 3: Side view of the cylindrical plasmonic nanocircuit without OTL.

where $J_{0}$ and $J_{1}$ are the Bessel functions of first kind of orders zero and one, respectively, $\omega$ is the operating angular frequency, $k_{0}$ is the propagation constant in air, $\mu_{0}$ is the magnetic permeability of air, and $\varepsilon_{0}$ is its electrical permittivity of air.

The equation for the scattered electric field due the current on length $l$ of the nanocircuit is given by

$$
\begin{array}{r}
\bar{E}_{s}(\bar{r})=\frac{1}{j \omega \varepsilon_{0}}\left[k_{0}^{2} \int_{l} \bar{I} g(R) d l^{\prime}+\int_{l} \frac{d I}{d l^{\prime}} \nabla g(R) d l^{\prime}\right], \\
g(R)=\frac{e^{-j k_{0} R}}{4 \pi R}, R=\left|\bar{r}-\bar{r}^{\prime}\right|,
\end{array}
$$

where $g(R)$ is Green's function in free space and $R$ is the distance between the observation point $\bar{r}$ (field point) and the source $\bar{r}^{\prime}$ (source point on the conductor axis).

Substituting (4) in (1), one obtains the integral equation of the problem:

$$
\begin{aligned}
& \left(\frac{1}{j \omega \varepsilon_{0}}\left[k_{0}^{2} \int_{l} \bar{I} g(R) d l^{\prime}+\int_{l} \frac{d I}{d l^{\prime}} \nabla g(R) d l^{\prime}\right]+\bar{E}_{i}\right) \cdot \bar{a}_{l} \\
& \quad=Z_{s} I .
\end{aligned}
$$

Figure 4 illustrates the discretization used in the MoM model of the nanocircuit in top view of plane $x y$, where $N_{L}$, $N_{h d}, N_{W e}$, and $N_{H e}$ are the numbers of the straight segments along $L, h_{d}, W_{e}$, and $H_{e}$, respectively. The discretization is uniform in these dimensions. The length of each segment is $\Delta L=L / N_{L}, \Delta h_{d}=h_{d} / N_{h d}, \Delta W_{e}=W_{e} / N_{W e}$, and $\Delta H_{e}=H_{e} / N_{H e}$, respectively. The gap of the voltage source is $d$ and it has two vertical segments in the source section (dashed segment in Figure 4). The total number of straight segments of the optical nanoantenna is $N_{t}=2 N_{h d}+2 N_{L}+$ $2 N_{W e}+2 N_{H e}+2$. The convergence criterion (stability) used to select $N_{L}, N_{h d}, N_{W e}$, and $N_{H e}$ is the maximum value that satisfies the constraints $\Delta L>2 a_{L}, \Delta h_{d}>2 a_{d}, \Delta W_{e}>$ $2 a_{e}$, and $\Delta H_{e}>2 a_{e}$. Therefore, we ensure the stability of the method considering the linear approximation of the current. The current distribution in each segment of Figure 4 is approximated by sinusoidal basis functions [16] and the number of these functions is $N=N_{t}-1$. To determine each of these $N$ triangular sinusoidal currents there is $N$ rectangular pulse test functions with unitary amplitude [16]. Thus, we have the following system of linear equations:

$$
V_{m}=Z_{s} I_{m} \Delta_{m}-\sum_{n=1}^{N} Z_{m n} I_{n}, \quad m=1,2, \ldots, N,
$$

where the elements of $Z_{m n}$ represent the mutual impedance between the sinusoidal current elements $m$ and $n$ [16], $V_{m}$ is the voltage in each segment $m$ which is different from zero only at the position of the voltage source, and $\Delta_{m}=$ $1 / 2\left(\Delta l_{m}+\Delta l_{m+1}\right)$. The solution of system (6) gives the current along the nanocircuit, which allows one calculation of various parameters presented in Section 3.

\section{Numerical Results}

In this section, we discuss firstly the accuracy of the developed MoM code comparing our results with experimental 


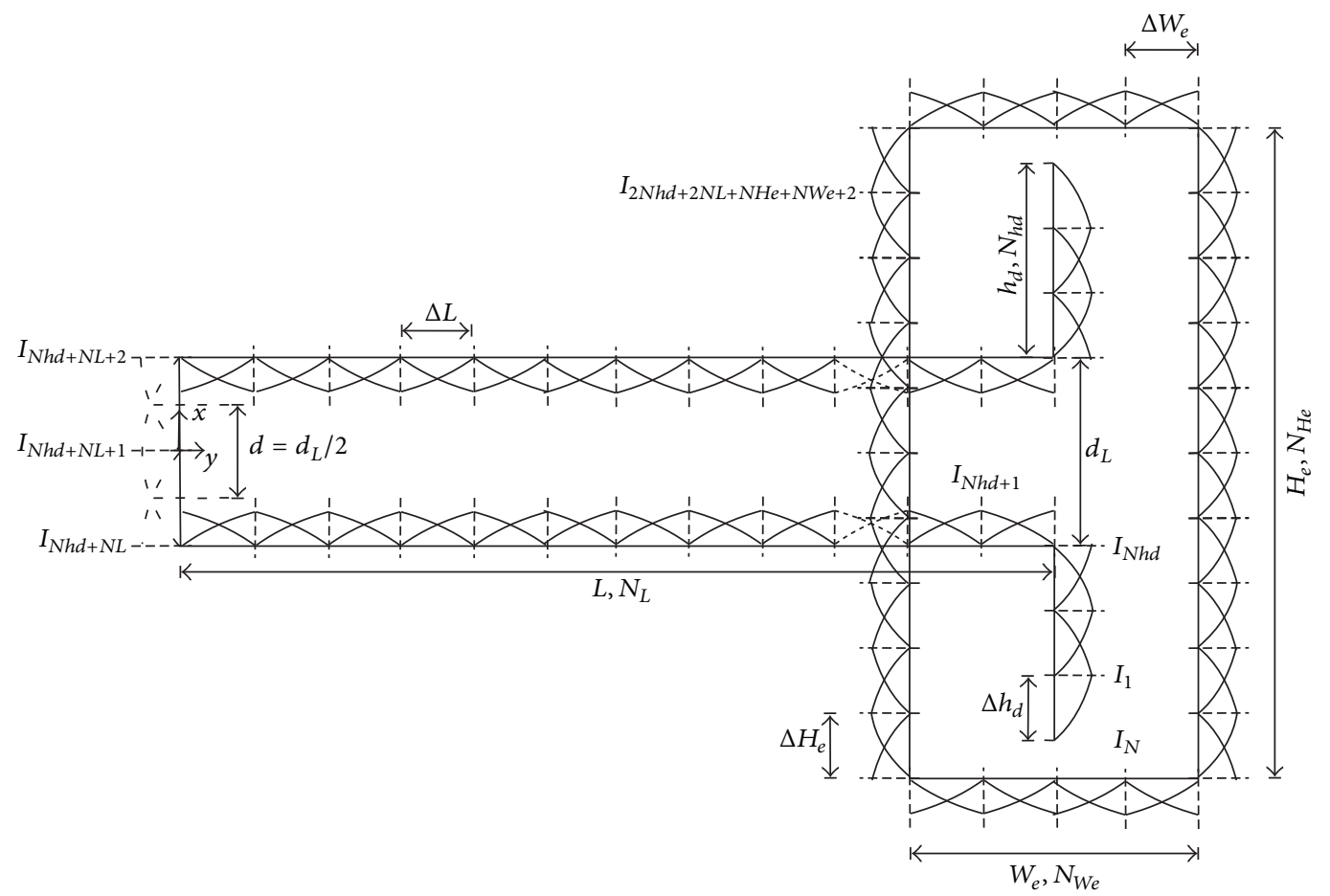

FIGURE 4: Discretization of nanocircuit, top view ( $z$-axis) for application of MoM.

data published in literature, then we analyze the nanoantenna separately from the nanocircuit, and, later on, the complete circuit will be investigated. Matlab codes were developed from the mathematical model presented in Section 2. The numerical results of the nanoantenna and nanocircuit are presented for the frequency range of $100-400 \mathrm{THz}$ and for a voltage source $\left(V_{s}\right)$ of $1 \mathrm{~V}$. In addition, some results were simulated in the Comsol Multiphysics software [17].

3.1. Experimental Validation of MoM Model. In order to verify the accuracy of our MoM code, this section shows a comparison between a cylindrical gold nanorod (Figure 5) simulated through MoM and an experimental arrangement of rectangular gold nanorods spaced by $5 \mu \mathrm{m}$ from one another presented in [20]. This comparison is valid because the interaction of the arrangement is minimal for the spacing of $5 \mu \mathrm{m}$ [20]. Both antennas are excited by a plane wave of magnitude $E_{0}$. The length of the cylindrical nanorod ( $h=$ $1030 \mathrm{~nm}$ ) equals that of the rectangular nanorod, the radius of the cylindrical nanorod $\left(a_{d}\right)$ is found by making the crosssectional area of the cylindrical nanorod equal to the crosssectional area of the rectangular nanorod (product of height $(100 \mathrm{~nm})$ and width $(90 \mathrm{~nm}))$, and the relative permittivity of the medium of the cylindrical nanorod is the mean between the permittivities of the substrate layers of the experimental nanorod arrangement.

Among the various experimental results [20], the measured result of the extinction cross section $\left(\sigma_{\text {ext }}\right)$ was chosen for comparison (Figure 5). We can assert that this parameter in MoM is the sum of the cross sections due to absorption $\left(\sigma_{\mathrm{abs}}\right)$ and scattering $\left(\sigma_{\mathrm{sca}}\right)$; that is, $\sigma_{\text {ext }}=\sigma_{\mathrm{abs}}+\sigma_{\text {sca }}$, with

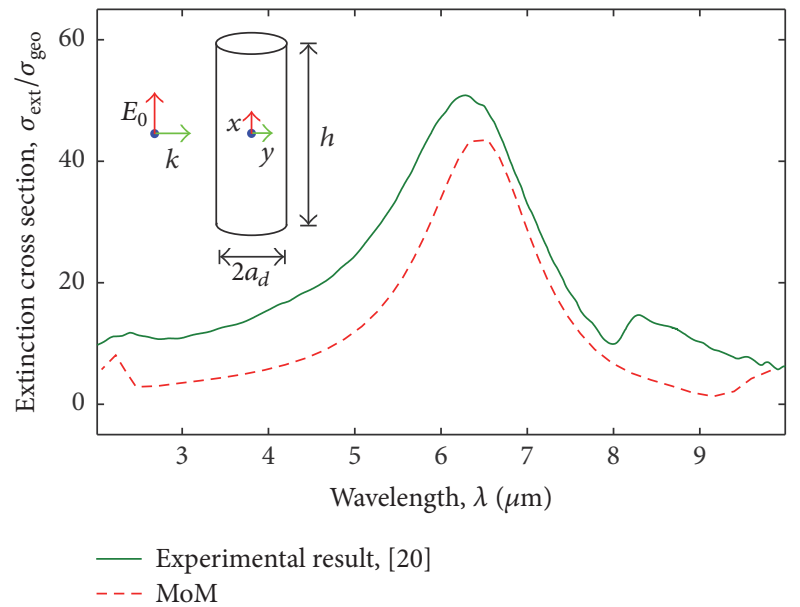

FIGURE 5: Extinction cross section normalized by the area of the geometrical cross section $\left(\sigma_{\text {ext }} / \sigma_{\text {geo }}\right)$ as a function of wavelength $\lambda$. The green solid line shows the results measured for a gold nanorod arrangement excited by a plane wave, which can be found in Figure 4 of [20]. The red dashed line shows the simulated result for a gold nanorod excited by a plane wave with $h=400 \mathrm{~nm}, a_{d}=47.5 \mathrm{~nm}$, and $N_{t}=10$.

$\sigma_{\mathrm{abs}}=P_{\mathrm{dis}} / S_{\mathrm{inc}}$ and $\sigma_{\mathrm{sca}}=P_{\mathrm{sca}} / S_{\mathrm{inc}}$, where $P_{\mathrm{dis}}$ and $P_{\mathrm{sca}}$ are, respectively, the dissipated power and scattered power and $S_{\text {inc }}$ is the Poynting vector of incident plane wave. Figure 5 shows the extinction cross section $\left(\sigma_{\text {ext }}\right)$ normalized by the area of the geometric cross section $\left(\sigma_{\text {geo }}\right)$ of the rectangular nanorod. This result shows that the MoM model produces outputs which are in a good agreement with the experimental 


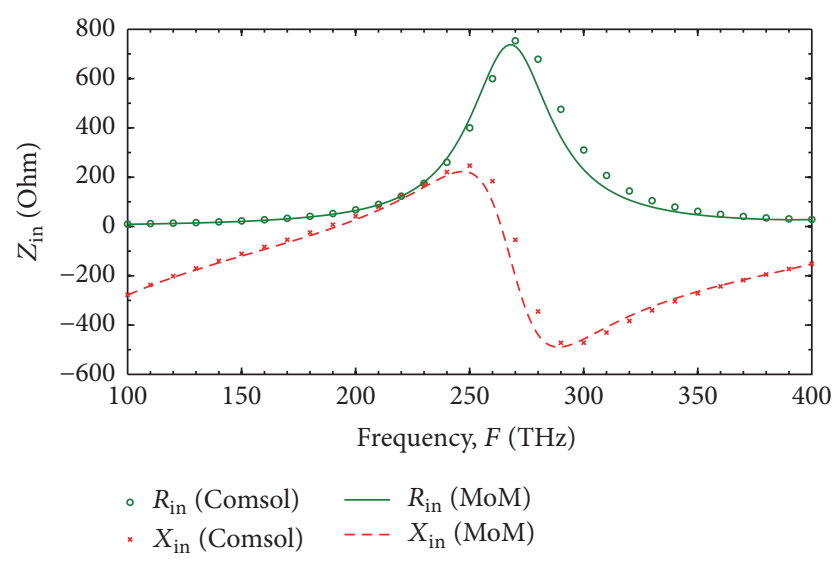

FIGURE 6: Input impedance of the electric nanodipole as a function of frequency.

result for the rectangular nanorods. The differences between results are due the approximation of nanorod diameter, equivalent relative permittivity of the medium, the linear approximation of the cylindrical nanorod with 9 base functions $(N=$ 9), and the array effect in far field that is not considered in MoM model.

3.2. Broadband Nanoantenna Analysis. Initially, the numerical results for a specific geometry with $h=220 \mathrm{~nm}, a_{d}=$ $20 \mathrm{~nm}, d=20 \mathrm{~nm}, d_{W}=50 \mathrm{~nm}, d_{H}=20 \mathrm{~nm}, a_{e}=20 \mathrm{~nm}$, $W_{e}=2 a_{e}+2 a_{d}+2 d_{W}, H_{e}=2 h+d+2 a_{e}+2 d_{H}, N_{h}=5, N_{H e}=13$, $N_{W e}=4$, and $N_{t}=46$ are shown. The first result obtained is the input impedance $\left(Z_{\text {in }}=R_{\text {in }}+j X_{\text {in }}\right)$ presented in Figure 6 for the case of isolated electrical nanodipole (i.e., without the loop) and this result is compared with the simulation by Comsol. It is noticeable in Figure 6 that the two methods produce values with good agreement for the used frequency range. As seen in the figure, the first resonant frequency for the MoM is $191.9 \mathrm{THz}$ and for the Comsol it is $187.7 \mathrm{THz}$, and the second resonant frequency for the MoM is $263.8 \mathrm{THz}$ and for the Comsol it is $267.7 \mathrm{THz}$.

Figure 7 also shows the input impedance $\left(Z_{\text {in }}=R_{\text {in }}+\right.$ $j X_{\text {in }}$ ) calculated by MoM and Comsol, but for the case of composed nanoantenna. One can note a good agreement between the two methods. It can be seen that the electromagnetic coupling between the dipole antenna and the loop antenna modifies the input impedance of the nanoantenna in comparison with the isolated electric dipole (Figure 6). It is further noted that the resonances are moved to the lower frequencies. The first resonant frequency $\left(F_{\lambda / 2}\right)$ for the MoM is $185.1 \mathrm{THz}$ and for the Comsol is $174.4 \mathrm{THz}$ and the second resonant frequency $\left(F_{\lambda}\right)$ for the MoM is $256.4 \mathrm{THz}$ and for the Comsol is $254.7 \mathrm{THz}$.

Figure 8 shows the results of calculation of the radiation efficiency and reflection coefficient obtained by MoM and Comsol for the isolated electrical nanodipole. The reflection coefficient is given by $\Gamma=\left|\left(Z_{\text {in }}-Z_{0}\right) /\left(Z_{\text {in }}+Z_{0}\right)\right|$, where $Z_{\text {in }}$ is input impedance of the nanoantenna and $Z_{0}$ is the characteristic impedance of the transmission line. The bandwidth is calculated by $B=200\left[\left(F_{s}-F_{i}\right) /\left(F_{s}+F_{i}\right)\right]$, where

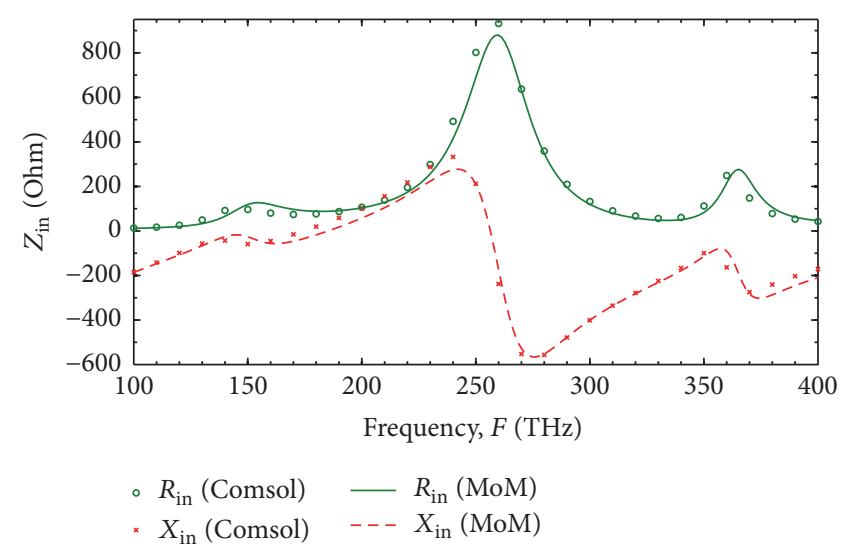

FIGURE 7: Input impedance of the composed nanoantenna as a function of frequency.

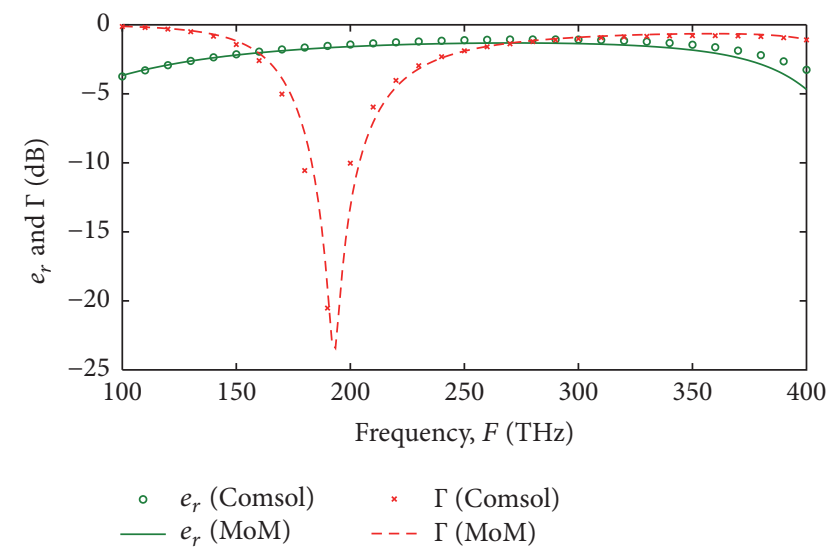

FIGURE 8: Radiation efficiency $\left(e_{r}\right)$ and reflection coefficient ( $\Gamma$ for $Z_{0}=60 \Omega$ ) of the isolated electrical nanodipole as a function of frequency, calculated by MoM and Comsol.

$F_{s}$ is the upper frequency and $F_{i}$ is the lower frequency at the level $-10 \mathrm{~dB}$ of the reflection coefficient.

We can note in Figure 8 that the maximum radiation efficiency calculated by the MoM and the Comsol is $-1.06 \mathrm{~dB}$ and $-1.32 \mathrm{~dB}$, respectively, occurring around the second resonant frequency. However, the best input impedance matching point occurs around the first resonant frequency, using $Z_{0}=60 \Omega$. Therefore, the maximum efficiency and good impedance matching are found at different frequencies. This occurs because the characteristic impedance of an OTL is not necessarily matched to the input impedance of nanodipole with maximum efficiency [12]. The calculation of the reflection coefficient has been accomplished considering the connection of a line with $Z_{0}=60 \Omega$, and for this impedance the final value obtained by the MoM was $B=10.1 \%$ and by Comsol $B=11.2 \%$. The results of $\Gamma$ show that the isolated dipole has a narrow bandwidth.

Figure 9 shows the radiation efficiency and reflection coefficient obtained by the MoM and Comsol for the composed nanoantenna shown in Figure 3 for $Z_{0}=90 \Omega$. One can see that insertion of the loop besides modifying the input impedance also changes the reflection coefficient causing 
TABLE 1: Results of parametric analysis of the composed nanoantenna (calculated through Comsol).

\begin{tabular}{|c|c|c|c|c|c|c|}
\hline & & \multicolumn{5}{|c|}{$d_{W}$} \\
\hline & & $30 \mathrm{~nm}$ & $40 \mathrm{~nm}$ & $50 \mathrm{~nm}$ & $60 \mathrm{~nm}$ & $70 \mathrm{~nm}$ \\
\hline \multirow{4}{*}{$d_{H}$} & \multirow{2}{*}{$10 \mathrm{~nm}$} & $B=35.4 \%$ & $B=36.8 \%$ & $B=38.3 \%$ & $B=40 \%$ & $B=42 \%$ \\
\hline & & $Z_{0}=85 \Omega$ & $Z_{0}=105 \Omega$ & $Z_{0}=100 \Omega$ & $Z_{0}=115 \Omega$ & $Z_{0}=120 \Omega$ \\
\hline & \multirow{2}{*}{$20 \mathrm{~nm}$} & $B=33.2 \%$ & $B=34.1 \%$ & $B=35.1 \%$ & $B=17.7 \%$ & $B=17.5 \%$ \\
\hline & & $Z_{0}=80 \Omega$ & $Z_{0}=85 \Omega$ & $Z_{0}=90 \Omega$ & $Z_{0}=80 \Omega$ & $Z_{0}=95 \Omega$ \\
\hline
\end{tabular}

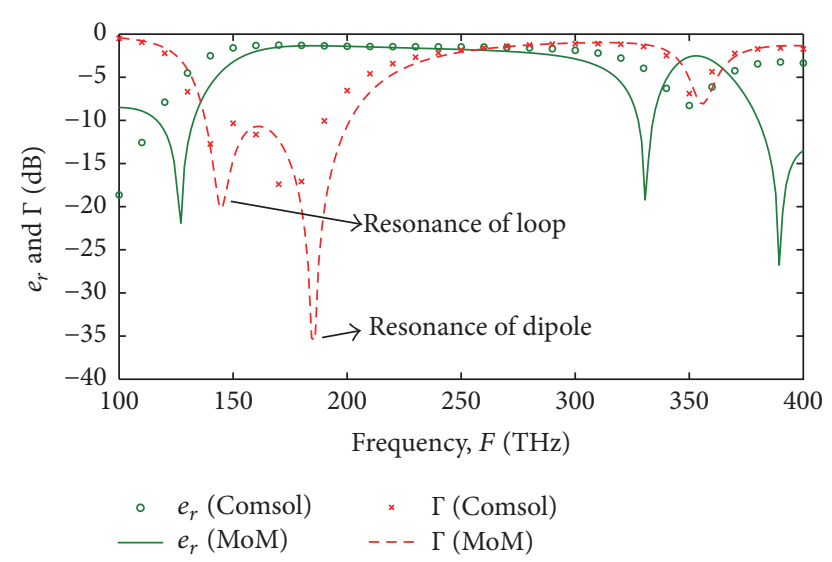

FIGURE 9: Radiation efficiency $\left(e_{r}\right)$ and reflection coefficient $(\Gamma$ for $Z_{0}=90 \Omega$ ) of the composed nanoantenna as a function of frequency, calculated through MoM and Comsol.

increase of the bandwidth of the nanoantenna to $37.1 \%$ by MoM and $35.1 \%$ by Comsol. The increased bandwidth occurs because of the inductive energy of the loop compensating the capacitive energy of the dipole, making an overlapping of different resonances of loop and dipole, which produces greater bandwidth [21], similar to that occuring with the combination of electrical and magnetic small dipoles in [15]. We can observe also the resonance of the loop (near $F \cong 145 \mathrm{THz}$ ) and the resonance of the dipole (near $F \cong 184 \mathrm{THz}$ ). In the frequency range between 150 and $280 \mathrm{THz}$ the radiation efficiency remains almost constant reaching the maximum value of $-1.276 \mathrm{~dB}$ by the Comsol simulation and $-1.35 \mathrm{~dB}$ for the MoM simulation. Thus we have a broadband antenna with high efficiency and with a possibility of achieving a good impedance matching with an optical transmission line (OTL) of $90 \Omega$.

Figure 10 shows the 3D far field gain radiation pattern for the isolated dipole and the composed nanoantenna. The diagram was calculated by far field approximation in MoM model; however, it could be calculated, for example, by ANSYS HFSS [22, 23] or Comsol [17]. The frequencies on these diagrams correspond to the set of central frequencies $\left(F_{c}=\left(F_{s}+F_{i}\right) / 2\right)$ of Figures 8 and 9 on $-10 \mathrm{~dB}$ level. The shape of the gain diagram of the isolated dipole (Figure 10) is approximately the same as for a small radiofrequency and microwave dipole. The maximum gain of the isolated dipole equals 1 for $F_{c}=194.97 \mathrm{THz}$ and the maximum gain of the composed antenna is equal to 1.4 for $F_{c}=170.85 \mathrm{THz}$. The
3D far field gain radiation pattern for other geometrical parameters and frequencies $(100-400 \mathrm{THz})$ of the composed nanoantenna is not shown in the article, since the shape of the diagram is approximately the same.

Table 1 shows a parametric analysis of the composed nanoantenna for $d_{W}=40,50,60$, and $70 \mathrm{~nm}$ and $d_{H}=10$ and $20 \mathrm{~nm}$, respectively, varying only the parameters $d_{W}$ and $d_{H}$, and fixed parameters $h=220 \mathrm{~nm}, a_{d}=20 \mathrm{~nm}, d=20 \mathrm{~nm}$, and $a_{e}=20 \mathrm{~nm}$. In this table the values of the impedance of the transmission line which maximize the bandwidth for each simulated antenna geometry are given. For these geometries, the input impedance is shown in Figures 11 and 12. In Figures 13 and 14, the radiation efficiency and the reflection coefficient are calculated by Comsol.

The results of Figures 11 and 12 show that if $d_{W}$ increases the resonance frequencies decrease, this can be better observed in the case of the second resonant frequency. This behavior is a characteristic of microwave antennas. This dependence of the resonances is similar to the variation of resonances of nanodipoles fed by a voltage source [24]. Furthermore, the maximum value of $R_{\text {in }}$ decreases. Regarding the results of Figures 13 and 14, the bandwidth and the radiation efficiency increased with the increase of $d_{W}$; however smaller values of $\Gamma$ are obtained with the decrease in $d_{W}$; that is, there is an improvement in impedance matching. Also, with the increase of $d_{H}$, bandwidth and efficiency decrease, and $\Gamma$ grows causing a decrease in input impedance matching. The maximum efficiency and a good input impedance matching occur at the same frequencies for the cases of broader bandwidths, as shown by the marked points in the figures.

In the next section, we consider an OTL connected to the composed nanoantenna for a quantitative analysis of the impedance matching of the OTL with the nanoantenna.

3.3. Analysis of Broadband Nanoantenna Connected to Optical Transmission Line. Figure 15 shows the current distribution along the nanocircuit for the frequencies of 100, 200, and $300 \mathrm{THz}$, for the following parameters: $h_{d}=200 \mathrm{~nm}, L=$ $1200 \mathrm{~nm}, a_{d}=a_{L}=a_{e}=20 \mathrm{~nm}, d_{L}=60 \mathrm{~nm}, d=d_{L}-2 a_{L}$, $d_{e}=50 \mathrm{~nm}, d_{W}=50 \mathrm{~nm}, d_{H}=20 \mathrm{~nm}, N_{h}=5, N_{W e}=4, N_{H e}$ $=13, N_{L}=29$, and $N_{t}=104$. In the figure, the circle points identify the currents of each section of the nanocircuit. The points to the left of 1 and between 4 and 5 are the currents in the electrical nanodipole, the ones between 1 and 2 and 3 and 4 are the currents of the OTL, those between 2 and 3 represent the current of the source, and finally those between 5 and 6,6 and 7, 7 and 8 and the right of 8 are the currents of 

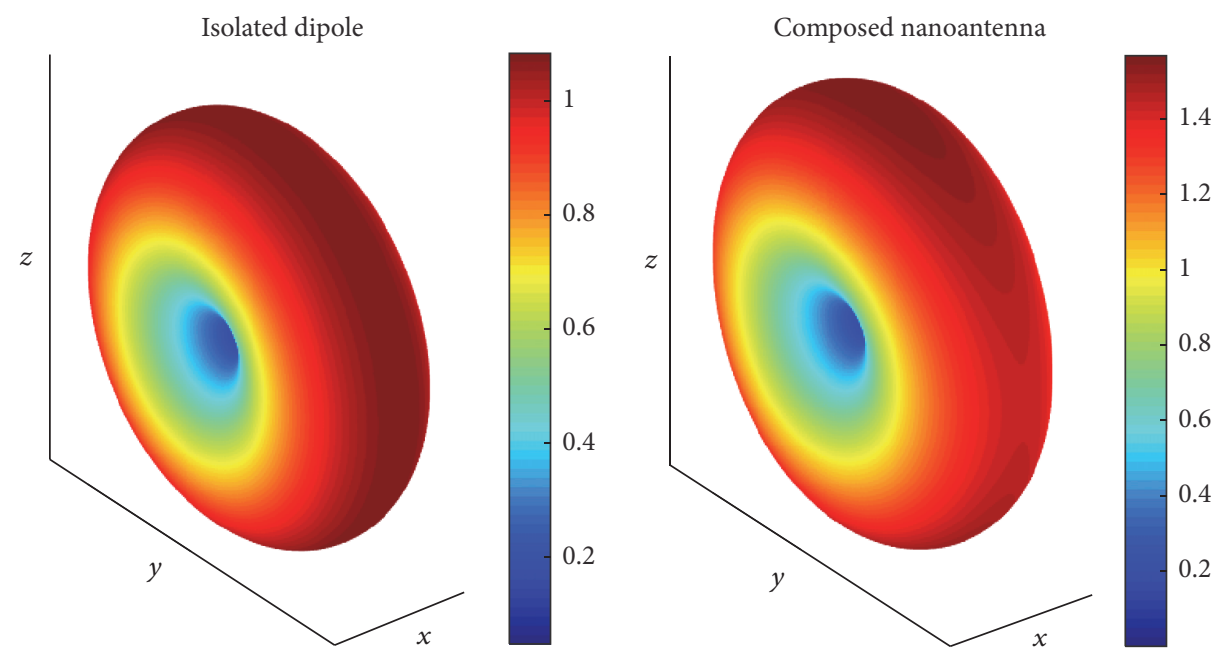

FIGURE 10: 3D far field gain radiation pattern of the isolated dipole and composed antenna for the central frequencies $194.97 \mathrm{and} 170.85 \mathrm{THz}$, respectively.
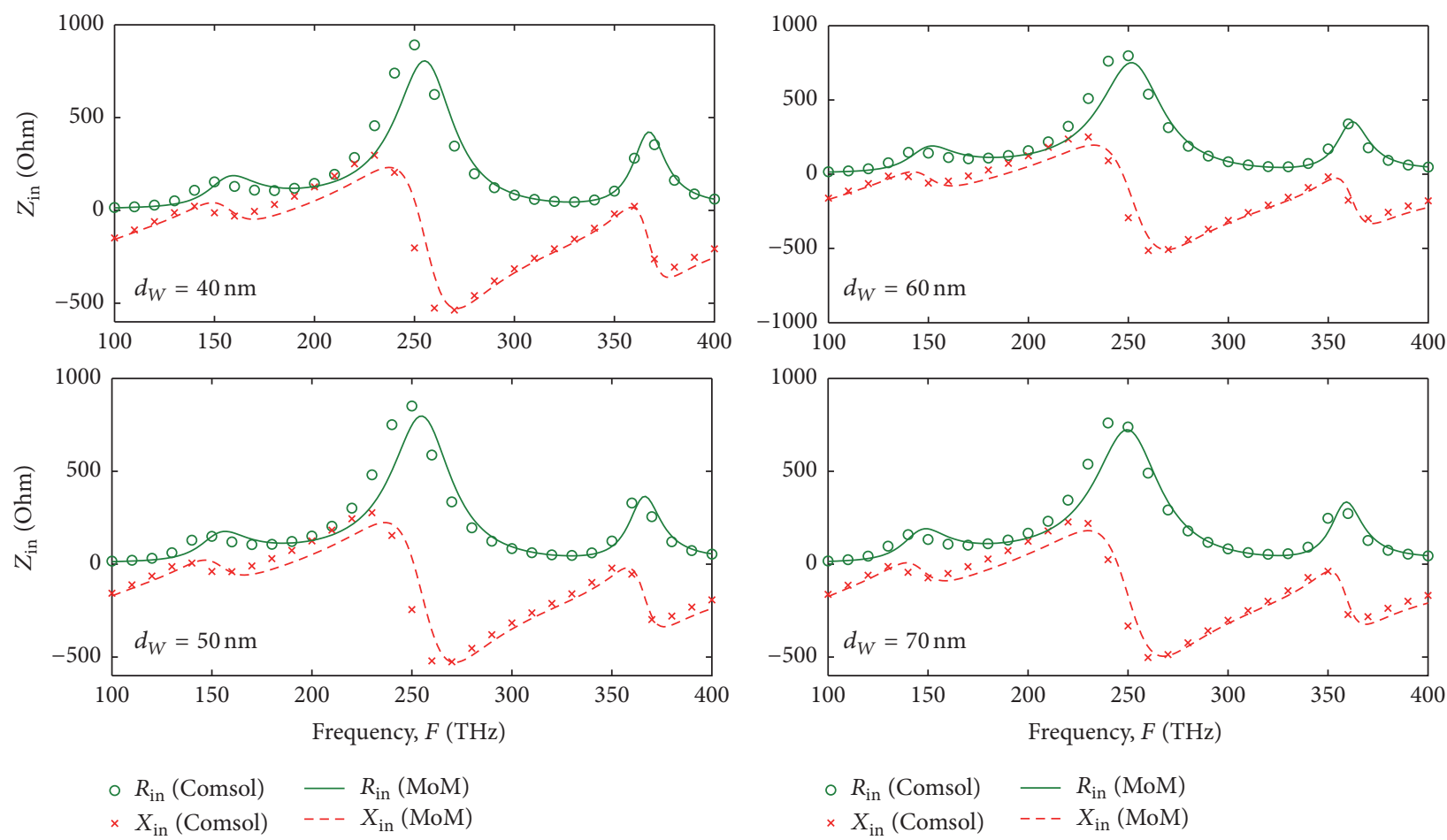

FIGURE 11: Input impedance of the composed nanoantenna for $d_{W}=40,50,60$, and $70 \mathrm{~nm}$ and $d_{H}=10 \mathrm{~nm}$.

the loop. One can see that while the frequency increases the attenuation of the current in the OTL increases, showing that at optical frequencies the conduction losses are higher. The stationary pattern presented in the figure shows the mismatch of the impedance of the optical transmission line and the nanodipole. The same oscillatory behavior is observed in Figure 16, where the distribution of the normalized electric field near a parallel plane to the nanocircuit (for the same frequencies as in Figure 15) is shown. These fields were calculated for the plane $z=80 \mathrm{~nm}$. It is evident that the energy transferred from the source to the nanodipole is reduced with increasing of frequency.

Figure 17 shows the 3D far field gain radiation diagram for this nanocircuit for $F=100,200$, and $300 \mathrm{THz}$. The shape of these diagrams can be explained when we consider that the nanocircuit in Figure 2 acts as an array of two antennas spaced by the length $L$ of the OTL, wherein one is the dipole-loop antenna (right side of circuit) and the second is an equivalent dipole formed by the voltage source (left side of circuit), which also radiates. Thus, the resulting radiation pattern of 

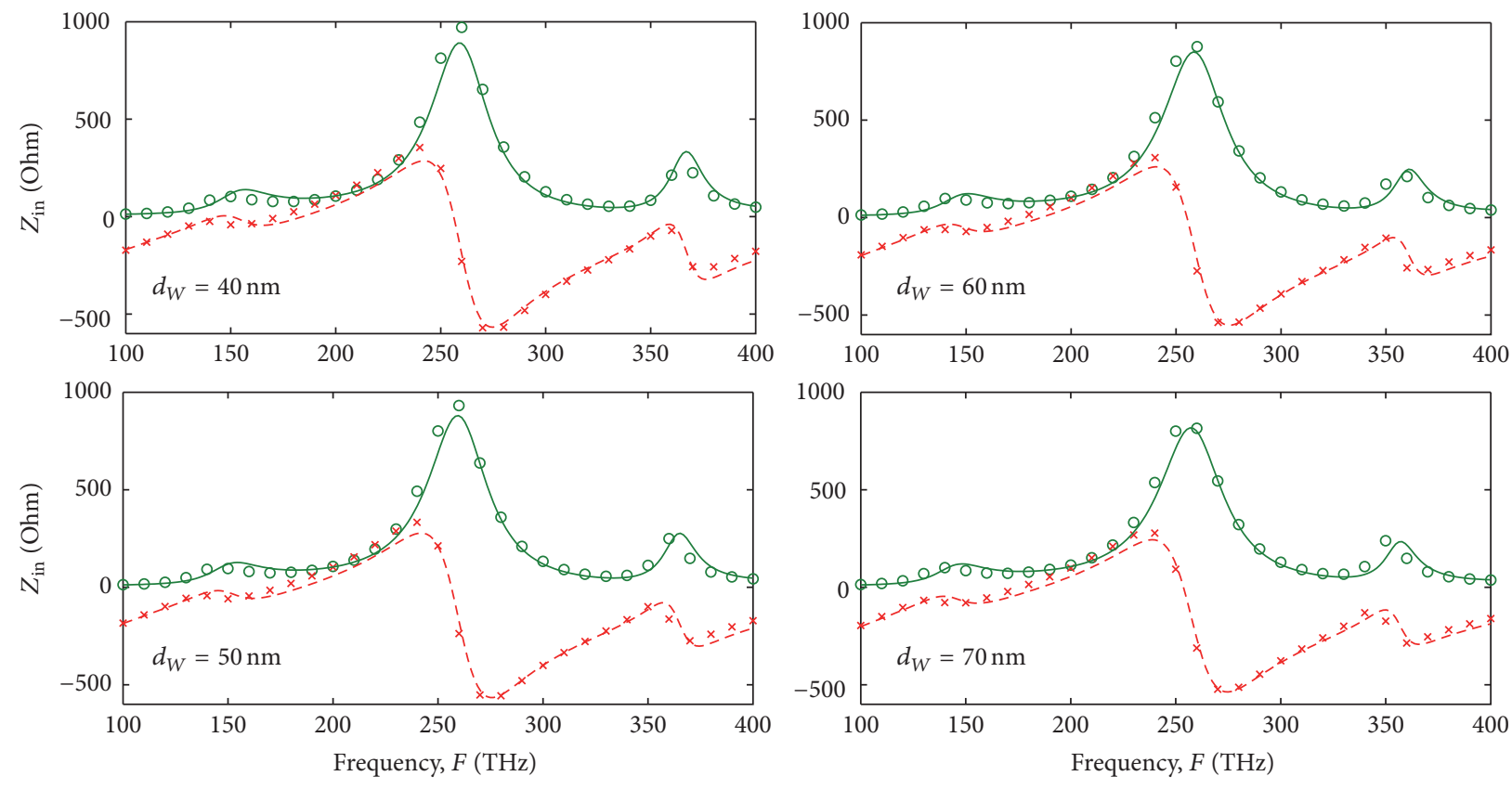

$\begin{array}{ll}\circ R_{\text {in }}(\text { Comsol }) & -R_{\text {in }}(\mathrm{MoM}) \\ \times X_{\text {in }}(\text { Comsol }) & ---X_{\text {in }}(\mathrm{MoM})\end{array}$

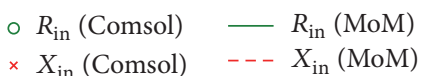

FIGURE 12: Input impedance of the composed nanoantenna for $d_{W}=40,50,60$, and $70 \mathrm{~nm}$ and $d_{H}=20 \mathrm{~nm}$.
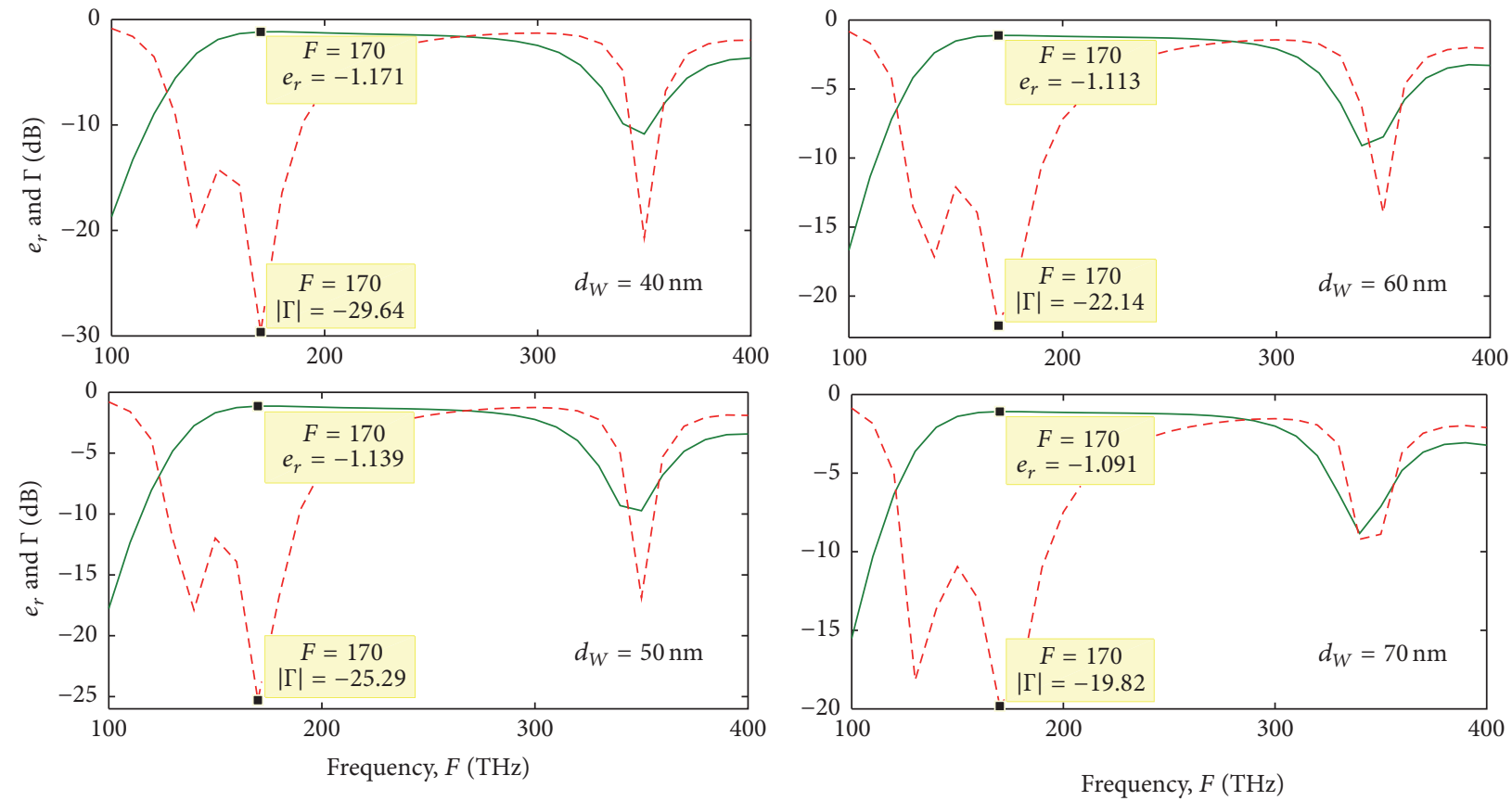

$$
\begin{aligned}
& -e_{r} \\
& --\Gamma
\end{aligned}
$$

FIGURE 13: Radiation efficiency $\left(e_{r}\right)$ and reflection coefficient $(\Gamma)$ of the composed nanoantenna as a function of the frequency calculated through Comsol with $d_{W}=40,50,60$, and $70 \mathrm{~nm}$ and $d_{H}=10 \mathrm{~nm}$. 

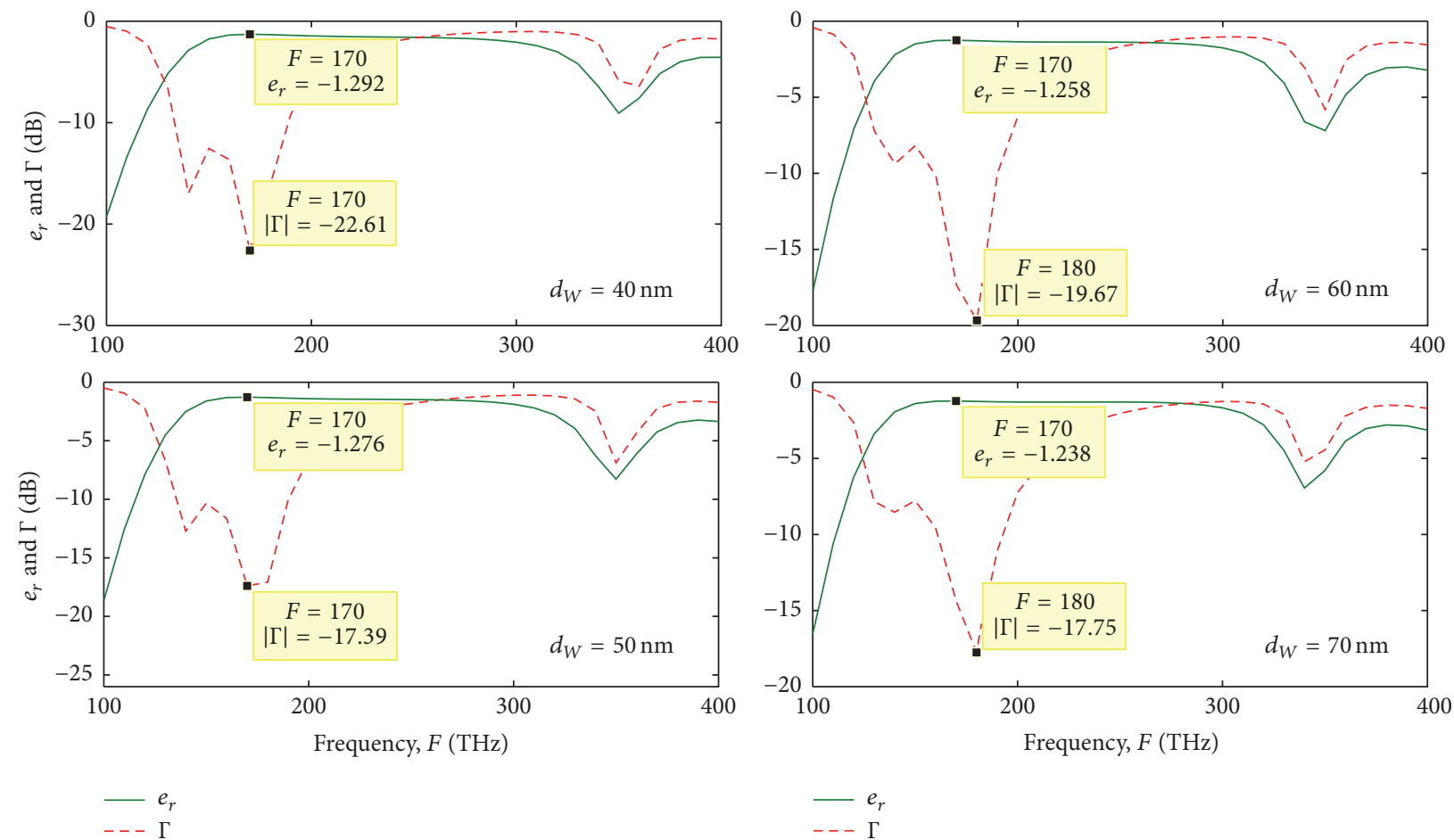

$-e_{r}$
$---\Gamma$

FIGURE 14: Radiation efficiency $\left(e_{r}\right)$ and reflection coefficient $(\Gamma)$ of the composed nanoantenna as a function of the frequency calculated through Comsol with $d_{W}=40,50,60$, and $70 \mathrm{~nm}$ and $d_{H}=20 \mathrm{~nm}$.

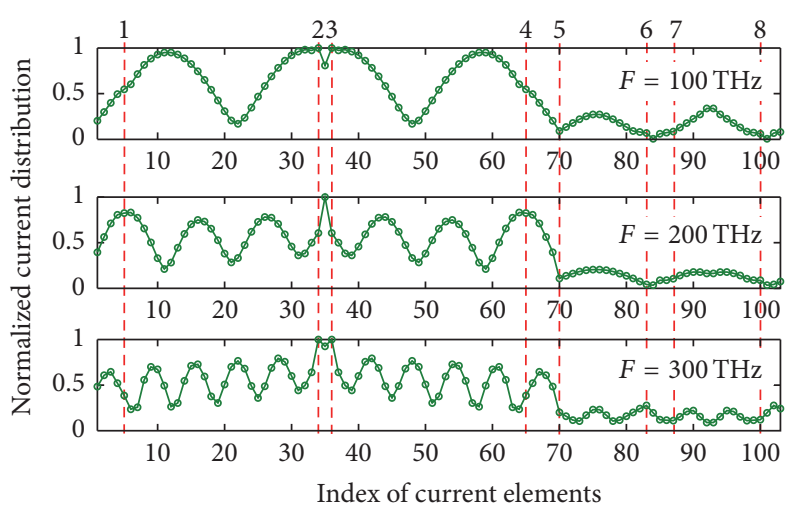

FIGURE 15: Normalized current distribution along the nanocircuit for $F=100,200$, and $300 \mathrm{THz}$, with the parameters $h_{d}=200 \mathrm{~nm}, L=$ $1200 \mathrm{~nm}, a_{d}=a_{L}=a_{e}=20 \mathrm{~nm}, d_{L}=60 \mathrm{~nm}, d_{e}=50 \mathrm{~nm}, d_{W}=50 \mathrm{~nm}, d_{H}=20 \mathrm{~nm}, N_{h d}=5, N_{W e}=4, N_{H e}=13, N_{L}=29$, and $N_{t}=104$.

this arrangement is the product of the radiation pattern of one element and the array factor, leading to the shape drawn in Figure 17. Furthermore, it can be noted that when frequency is increased the resulting pattern has more side lobes. Another observation in these diagrams is that the radiation intensity is higher in the $-z$ direction, because the loop element above the dipole acts as a reflector of waves.

To analyze the impedance matching of the OTL with the antenna, it is necessary to calculate the voltage reflection coefficient $\left(\left|\Gamma_{V}\right|=(\right.$ VSWR -1$) /($ VSWR +1$\left.)\right)$ as previously done in $[13,14,16]$, doing the approximate calculation of the voltage standing wave ratio (VSWR) near the electric nanodipole, VSWR $=I_{\max } / I_{\min }, I_{\max }$ is the maximum current magnitude, and $I_{\min }$ is the minimum current magnitude. The results of calculations are shown in Figure 18 for $\left|\Gamma_{V}\right|$ as a function of frequency for the nanocircuit with the loop and without it. In the figure the minimum points $\left|\Gamma_{V}\right|$ are highlighted. They are $-7.6 \mathrm{~dB}$ and $-13.53 \mathrm{~dB}$, at $F \cong$ $157.3 \mathrm{THz}$ and $F \cong 383.4 \mathrm{THz}$, respectively, for the case of the nanocircuit with the loop. This figure shows that the voltage reflection coefficient decreases with the addition of the loop into the optical nanocircuit near these frequencies 


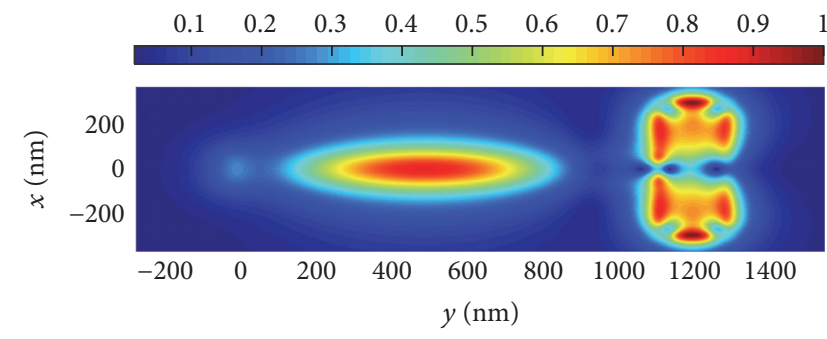

(a)

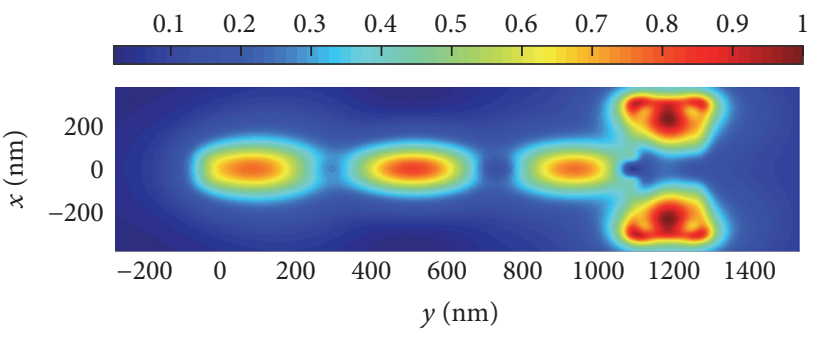

(b)

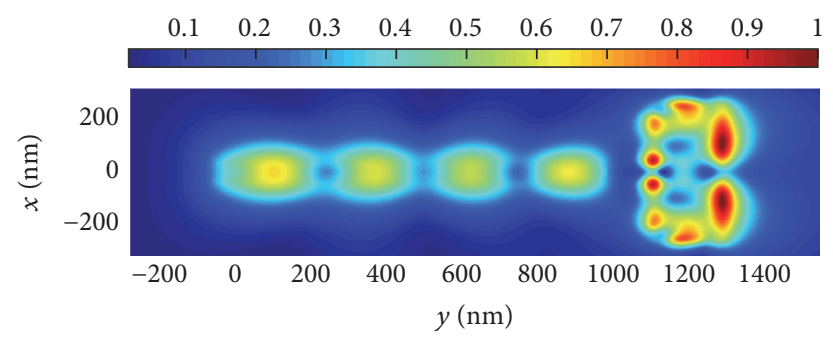

(c)

FIGURE 16: The electric field distribution in the plane $z=80 \mathrm{~nm}, F=100 \mathrm{THz}$ (a), $200 \mathrm{THz}$ (b), and $300 \mathrm{THz}$ (c), the parameters $h_{d}=200 \mathrm{~nm}$, $L=1200 \mathrm{~nm}, a_{d}=a_{L}=a_{e}=20 \mathrm{~nm}, d_{L}=60 \mathrm{~nm}, d_{e}=50 \mathrm{~nm}, d_{W}=50 \mathrm{~nm}, d_{H}=20 \mathrm{~nm}, N_{h d}=5, N_{W e}=4, N_{H e}=13, N_{L}=29$, and $N_{t}=104$.
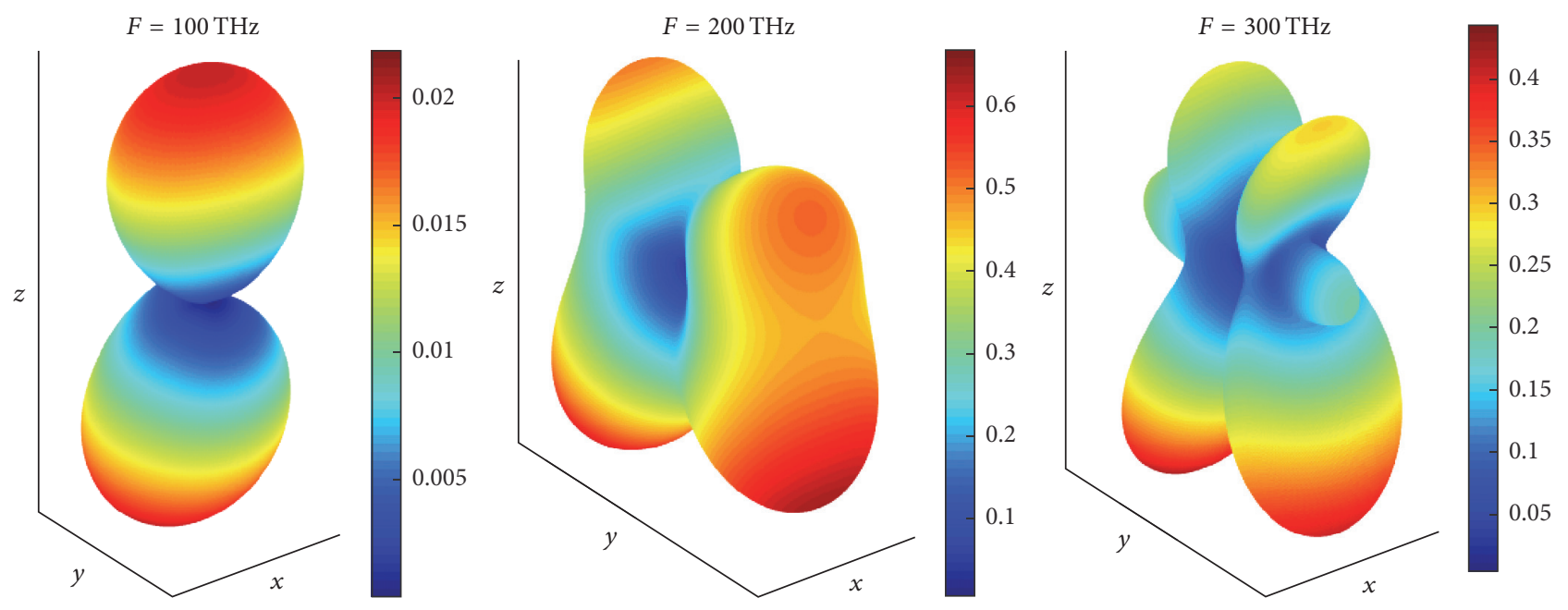

FIGURE 17: 3D far field gain radiation pattern of the circuit for $F=100,200$, and $300 \mathrm{THz}$.

( $F \cong 157.3$ and $F \cong 383.4 \mathrm{THz}$ ). Besides, this figure explains the stationary behavior of the current and field shown in Figures 15 and 16.

For the frequencies corresponding to these minimum voltage reflection coefficients for the case of the nanocircuit with loop, the current distribution in Figure 19 and normalized electrical field distribution in the plane $z=80 \mathrm{~nm}$ (Figure 20) are shown.

It can be observed in these figures (Figures 19 and 20) that with increasing of frequency the attenuation of current and electric field in the OTL increases due to conduction losses. It may be noted that for the frequency of $383.4 \mathrm{THz}$ there is a significant drop at the standing wave rate in relation to the frequency of $157.3 \mathrm{THz}$, which presents a decrease in reflection losses on the line. Thus, the point $\left|\Gamma_{V}\right|=-13.53 \mathrm{~dB}$ and $F=383.4 \mathrm{THz}$ correspond to a good impedance matching between the OTL and nanoantenna.

Figure 21 shows the 3D far field gain radiation pattern for the nanocircuit for $F=157.3$ and $383.4 \mathrm{THz}$. One observes again that the circuit in Figure 2 works like an array of two dipoles spaced by the length $L$ of the OTL. Also, the gain level of case $157.3 \mathrm{THz}$ is higher than that for $383.4 \mathrm{THz}$, which is in accordance with the higher current levels of Figure 19.

Finally, a parametric analysis of the voltage reflection coefficient is shown in Figures 22 and 23. For the simulations we fix the following parameters: the distance between the surfaces of the OTL $(D=20 \mathrm{~nm})$, the length of the electric dipole $(h=220 \mathrm{~nm})$, the radii $\left(a_{L}=20 \mathrm{~nm}, a_{d}=20 \mathrm{~nm}\right.$, and $\left.a_{e}=20 \mathrm{~nm}\right)$, and the length of the OTL $(L=1200 \mathrm{~nm})$, still varying the parameters $d_{W}\left(d_{W}=30,40\right.$, and $\left.50 \mathrm{~nm}\right)$ 


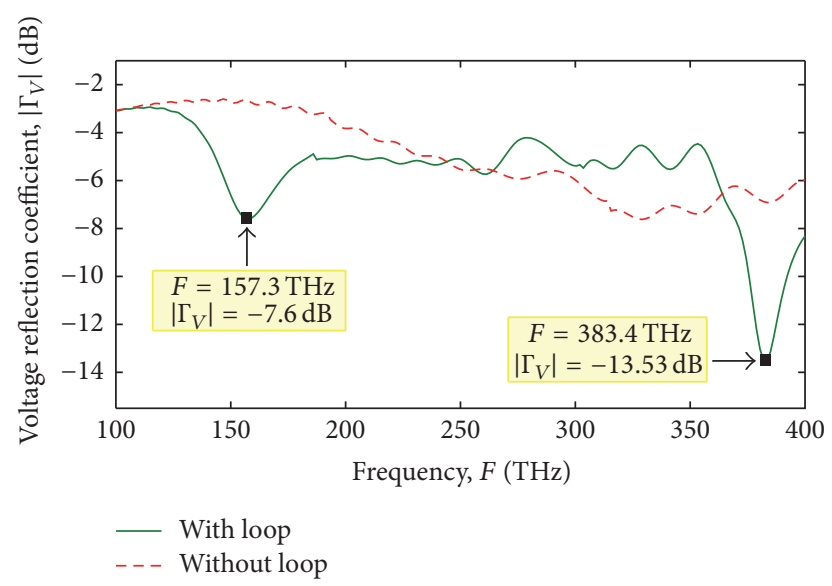

FIGURE 18: Voltage reflection coefficient as a function of frequency; the parameters are $h_{d}=200 \mathrm{~nm}, L=1200 \mathrm{~nm}, a_{d}=a_{L}=a_{e}=20 \mathrm{~nm}$, $d_{L}=60 \mathrm{~nm}, d_{e}=50 \mathrm{~nm}, d_{W}=50 \mathrm{~nm}, d_{H}=20 \mathrm{~nm}, N_{h d}=5, N_{W e}=$ $4, N_{H e}=13, N_{L}=29$, and $N_{t}=104$.

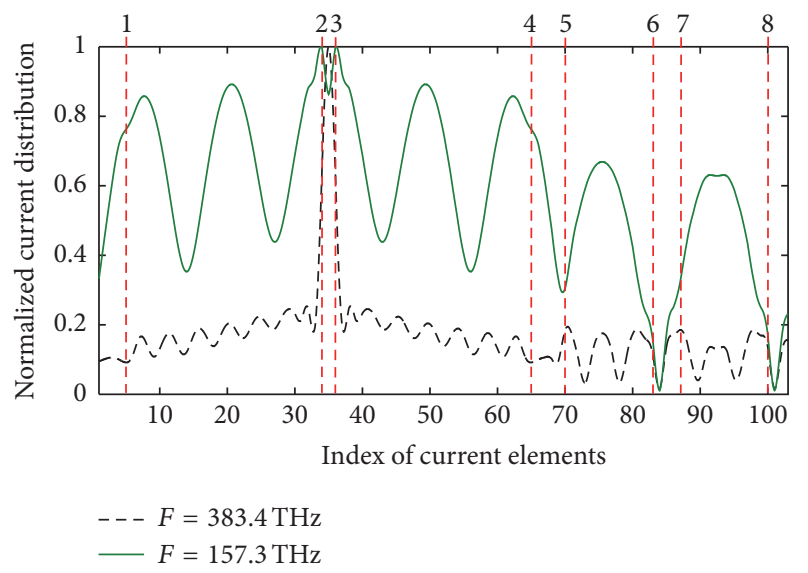

FIGURE 19: Current distribution in the nanocircuit for $F=157.3$ and $383.4 \mathrm{THz}$, for cases with reflection coefficient $\left|\Gamma_{V}\right|=-7.6$ and $-13.5 \mathrm{~dB}$, respectively.

and $d_{H}\left(d_{H}=10\right.$ and $\left.20 \mathrm{~nm}\right)$, that consequently change the width $\left(W_{e}+2 a_{e}\right)$ and the length $\left(H_{e}+2 a_{e}\right)$ of the loop. In Figures 22 and 23 we show the results for the voltage reflection coefficient without the loop. Analyzing these figures one comes to the following conclusions. In all simulated geometry of the circuit with the loop there is an improvement in comparison with the circuit without the loop regarding impedance matching at some points as can be seen in the figures. The second conclusion is that, for smaller values of $d_{W}$, the curves of the voltage reflection coefficient $\left(\left|\Gamma_{V}\right|\right)$ are shifted to the higher frequencies. This occurs because the impedance matching depends on the positions of the resonances of the nanoantenna that are shifted to higher frequencies for smaller lengths of $d_{W}$. In general, the best impedance matching is obtained for smaller values of $d_{W}$. This behavior can be explained by reduction of the reflection coefficient $\left(\left|\Gamma_{V}\right|\right)$ of the nanoantenna when $d_{W}$ decreases. But then, an increase on $d_{H}$ results in increased values of $\left|\Gamma_{V}\right|$. This occurs because the reflection coefficient of nanoantenna increases with the increase on $d_{H}$.

\section{Conclusions}

In this study, we analyzed one application of cylindrical broadband nanoantennas in plasmonic nanocircuits, where the circuit comprises a nanoantenna connected to a two-wire optical transmission line. The nanoantenna presents a combination of a loop and a dipole antenna. In the circuit modeling, the Lorentz-Drude model was used to represent the physical characteristics of the metal. The method of moments (MoM) was applied to find the solution of the $1 \mathrm{D}$ integral equation for the electric field with linear approximation of the longitudinal current, finite surface impedance to represent losses in the conductor, sinusoidal basis functions, and rectangular pulse for test functions. To validate the MoM model we compared a MoM simulation with an experimental result from [20]. In addition, some MoM simulations were compared with simulations carried out by the commercial package Comsol.

Initially, the nanoantenna was investigated separately from the nanocircuit and then the complete circuit was investigated. In relation to the study of the isolated nanoantenna, the results show that the electromagnetic coupling between the antennas (dipole and loop) modifies the input impedance and increases the bandwidth of nanoantenna in comparison with the isolated electrical dipole. The best result for the nanoantenna bandwidth is $42 \%$, and, in general, for all simulations, this bandwidth was within $33.2<B(\%) \leq$ 42. In addition, it can be observed that the bandwidth and radiation efficiency of the nanoantenna can be optimized through alterations of the geometry of the rectangular loop. Another important result is that radiation efficiency and good impedance matching depending on the geometry of the antenna and can be achieved in the same frequency region. This means that the suggested nanoantennas can be used in applications such as broadband plasmonic nanocircuits.

The nanocircuit which combines the nanoantenna and OTL was also investigated, focusing on the input impedance matching between the OTL and the nanoantenna. The obtained results showed that the overall voltage reflection coefficient decreases with the inclusion of the rectangular loop, reaching a value of approximately $-25 \mathrm{~dB}$. A good impedance matching can be achieved in different frequency bands tuning the nanocircuit operating frequency by varying the width and length of the rectangular loop. Another important result is the far field gain radiation pattern of the circuit, where the shape of the pattern suggests that the circuit behaves as an array of two antennas spaced by the length $L$ of the OTL.

The analyses presented in this paper may be useful as guidelines for the design of efficient plasmonic optical nanocircuits for applications in nanophotonics and nanoelectronics. 


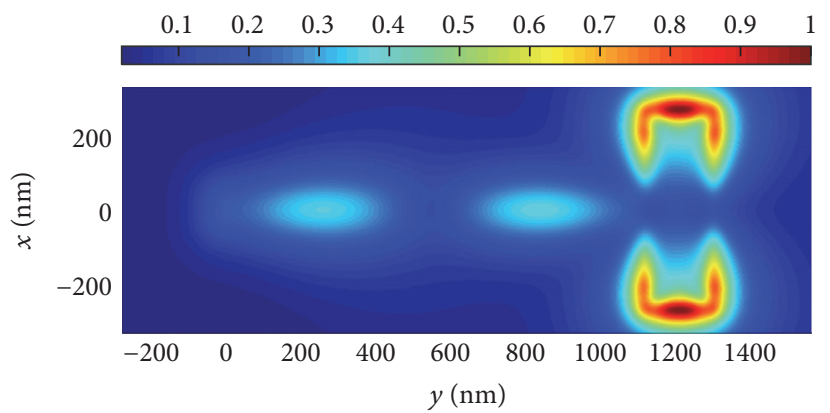

(a)

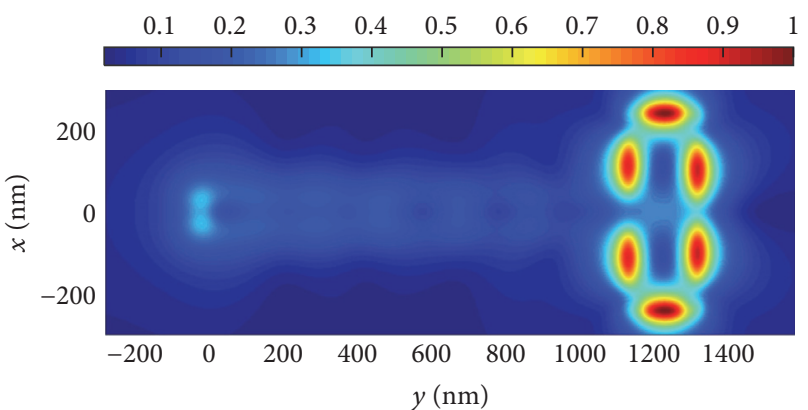

(b)

FiguRE 20: Electric field distribution in plane $z=80 \mathrm{~nm}$ for the frequencies $F=157.3$ (a) and 383.4 (b) THz, for cases with the reflection coefficient $\left|\Gamma_{V}\right|=-7.6$ and $-13.53 \mathrm{~dB}$, respectively.
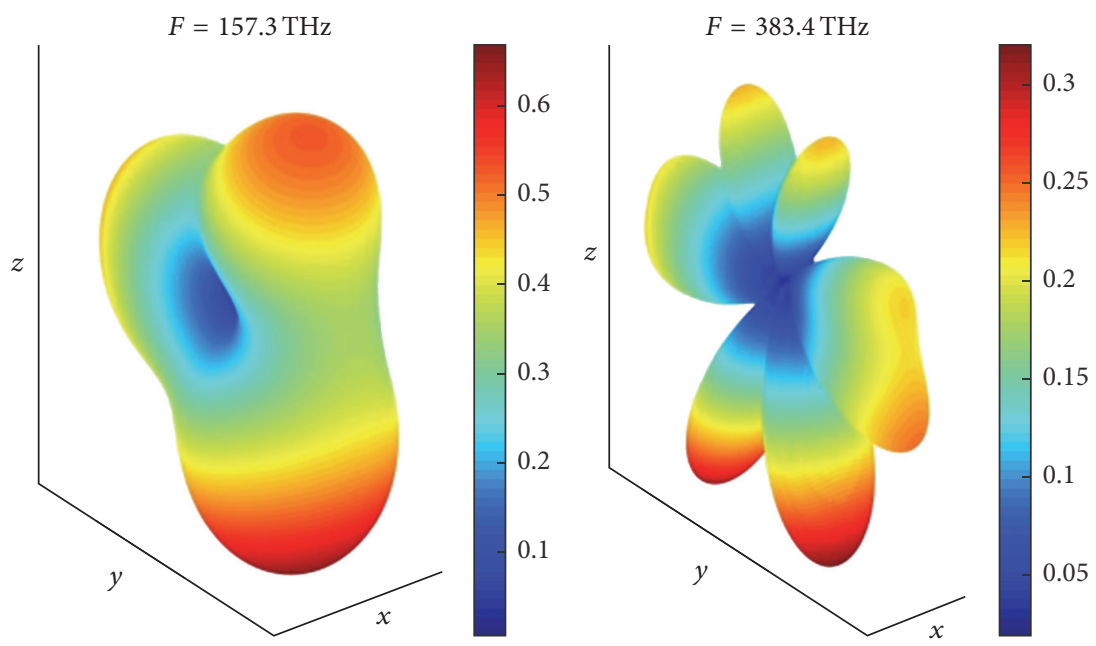

FIGURE 21: 3D far field gain radiation pattern of the circuit for $F=157.3$ and $383.4 \mathrm{THz}$.

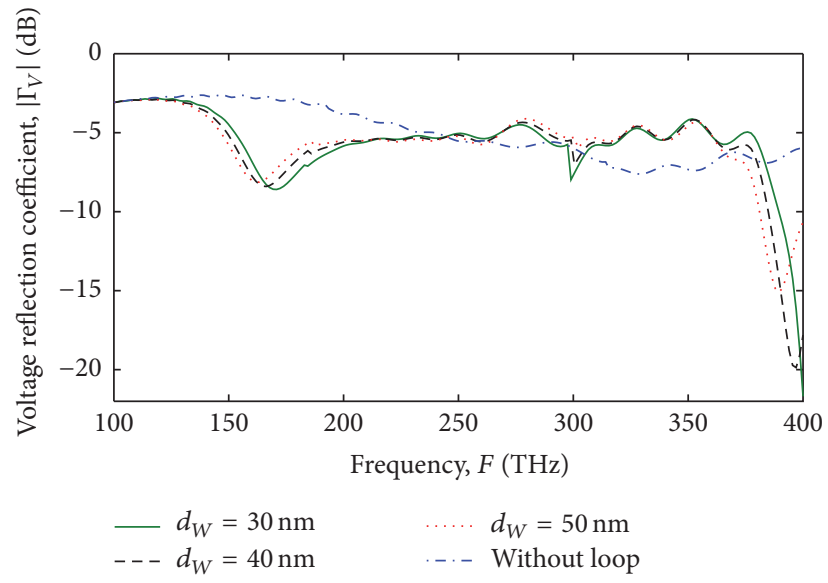

FIGURE 22: Voltage reflection coefficient $\left(\left|\Gamma_{V}\right|\right)$ with the loop near the dipole for different values of $d_{W}(30,40$, and $50 \mathrm{~nm})$ with $d_{H}=10 \mathrm{~nm}$ and also $\left|\Gamma_{V}\right|$ without the loop.

\section{Competing Interests}

The authors declare that there is no conflict of interests regarding the publication of this paper.

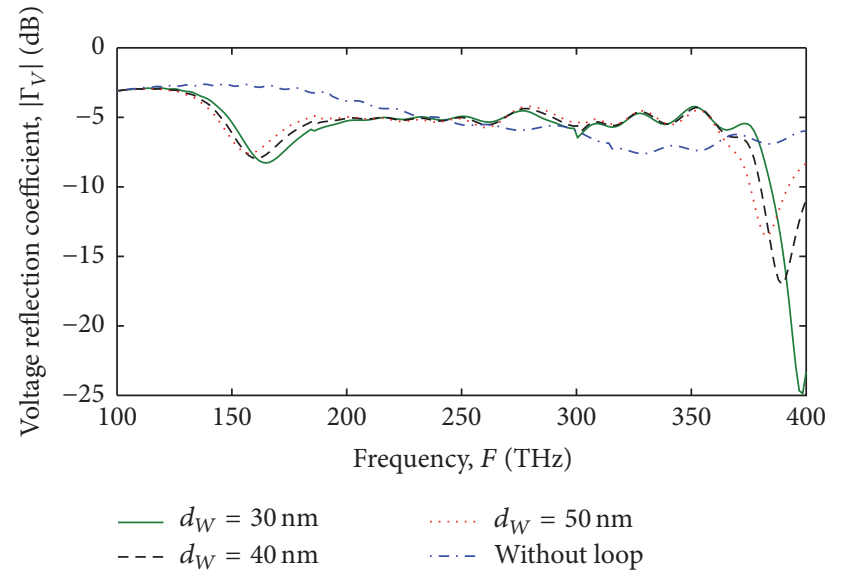

FIGURE 23: Voltage reflection coefficient $\left(\left|\Gamma_{V}\right|\right)$ with the loop near the dipole for different values of $d_{W}(30,40$, and $50 \mathrm{~nm})$ with $d_{H}=$ $20 \mathrm{~nm}$ and also $\left|\Gamma_{V}\right|$ without the loop.

\section{Acknowledgments}

This work was supported by the Brazilian agencies PROPESP/UFPA, CAPES, and CNPq. 


\section{References}

[1] P. Bharadwaj, B. Deutsch, and L. Novotny, "Optical Antennas," Advances in Optics and Photonics, vol. 1, no. 3, pp. 438-483, 2009.

[2] L. Novotny and N. Van Hulst, "Antennas for light," Nature Photonics, vol. 5, no. 2, pp. 83-90, 2011.

[3] K. Q. Le, "Broadband light trapping in thin organic photovoltaic cells using plasmonic resonant antennas," Journal of Applied Physics, vol. 114, no. 8, Article ID 084504, 2013.

[4] H. A. Atwater and A. Polman, "Plasmonics for improved photovoltaic devices," Nature Materials, vol. 9, no. 3, pp. 205213, 2010.

[5] R. M. Bakker, H.-K. Yuan, Z. Liu et al., "Enhanced localized fluorescence in plasmonic nanoantennae," Applied Physics Letters, vol. 92, no. 4, 2008.

[6] K. Li, L. Clime, B. Cui, and T. Veres, "Surface enhanced Raman scattering on long-range ordered noble-metal nanocrescent arrays," Nanotechnology, vol. 19, no. 14, Article ID 145305, 2008.

[7] R. Zhou, J. Ding, B. Arigong, Y. Lin, and H. Zhang, "Design of a new broadband monopole optical nano-antenna," Journal of Applied Physics, vol. 114, no. 18, Article ID 184305, 2013.

[8] M. Navarro-Cia and S. A. Maier, "Broad-band near-infrared plasmonic nanoantennas for higher harmonic generation," ACS Nano, vol. 6, no. 4, pp. 3537-3544, 2012.

[9] E. S. Ünlü, R. U. Tok, and K. Şendur, "Broadband plasmonic nanoantenna with an adjustable spectral response," Optics Express, vol. 19, no. 2, pp. 1000-1006, 2011.

[10] A. E. Miroshnichenko, I. S. Maksymov, A. R. Davoyan, C. Simovski, P. Belov, and Y. S. Kivshar, "An arrayed nanoantenna for broadband light emission and detection," Physica Status Solidi-Rapid Research Letters, vol. 5, no. 9, pp. 347-349, 2011.

[11] D. K. Gramotnev and S. I. Bozhevolnyi, "Plasmonics beyond the diffraction limit," Nature Photonics, vol. 4, no. 2, pp. 83-91, 2010.

[12] J.-S. Huang, T. Feichtner, P. Biagioni, and B. Hecht, "Impedance matching and emission properties of nanoantennas in an optical nanocircuit," Nano Letters, vol. 9, no. 5, pp. 1897-1902, 2009.

[13] K. Costa, V. Dmitriev, J. Souza, and G. Silvano, "Analysis of nanodipoles in optical nanocircuits fed by gaussian beam," International Journal of Antennas and Propagation, vol. 2014, Article ID 429425, 12 pages, 2014.

[14] K. Q. Da Costa, V. A. Dmitriev, and G. L. Silvano, "Impedance matching analysis of an optical nanocircuit fed by an aperture probe," Journal of Microwaves, Optoelectronics and Electromagnetic Applications, vol. 12, pp. si42-si56, 2013.

[15] K. Q. Da Costa and V. Dmitriev, "Combination of electric and magnetic dipoles with single-element feeding for broadband applications," Microwave and Optical Technology Letters, vol. 48, no. 1, pp. 8-12, 2006.

[16] K. Costa and V. Dmitriev, "Simple and efficient computational method to analyze cylindrical plasmonic nanoantennas," International Journal of Antennas and Propagation, vol. 2014, Article ID 675036, 8 pages, 2014.

[17] COMSOL Multiphysic 4.4a, COMSOL Inc., http://www.comsol .com.

[18] L. Novotny and B. Hecht, Principles of Nano-Optics, Cambridge University Press, Cambridge, UK, 2006.

[19] G. W. Hanson, "On the applicability of the surface impedance integral equation for optical and near infrared copper dipole antennas," IEEE Transactions on Antennas and Propagation, vol. 54, no. 12, pp. 3677-3685, 2006.
[20] D. Weber, P. Albella, P. Alonso-González et al., "Longitudinal and transverse coupling in infrared gold nanoantenna arrays: long range versus short range interaction regimes," Optics Express, vol. 19, no. 16, pp. 15047-15061, 2011.

[21] R. E. Collin and S. Rothschild, "Evaluation of Antenna Q," IEEE Transactions on Antennas and Propagation, vol. 12, no. 1, pp. 2327, 1964.

[22] L. Gong, K. Y. Chan, and R. Ramer, "A split-ring structures loaded SIW sectorial horn antenna," in Proceedings of the 5th IEEE-APS Topical Conference on Antennas and Propagation in Wireless Communications (APWC '15), pp. 349-350, Turin, Italy, September 2015.

[23] L. Gong, K. Y. Chan, and R. Ramer, "Substrate integrated waveguide $\mathrm{H}$-plane horn antenna with improved front-to-back ratio and reduced sidelobe level," IEEE Antennas and Wireless Propagation Letters, vol. 15, pp. 1835-1838, 2016.

[24] K. Q. Da Costa and V. Dmitriev, "Radiation and absorption properties of gold nanodipoles in transmitting mode," Microwave and Optical Technology Letters, vol. 57, no. 1, pp. 1-6, 2015. 


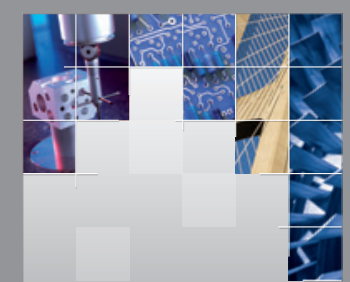

\section{Enfincering}
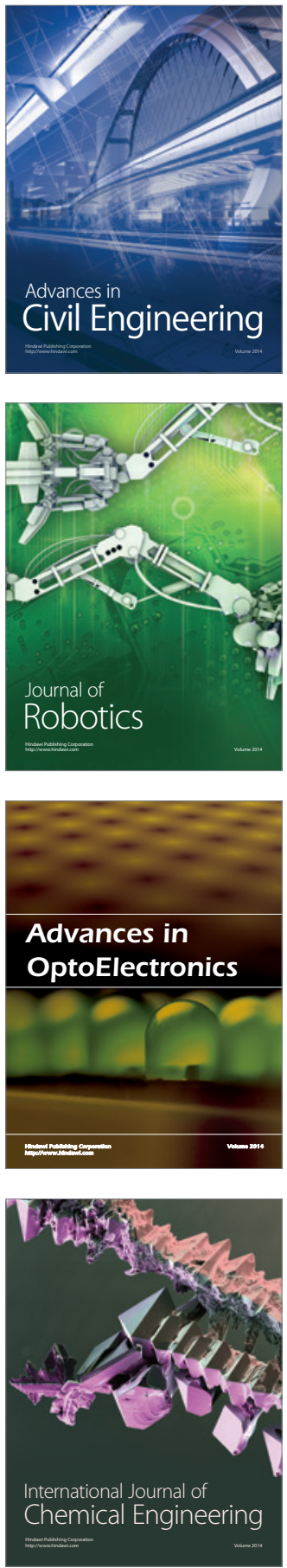

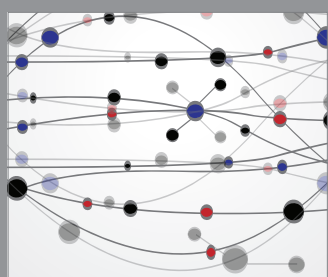

The Scientific World Journal

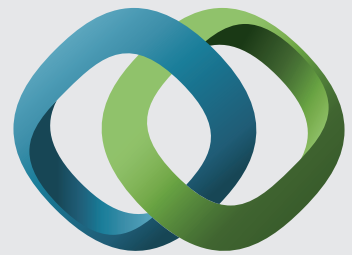

\section{Hindawi}

Submit your manuscripts at

https://www.hindawi.com
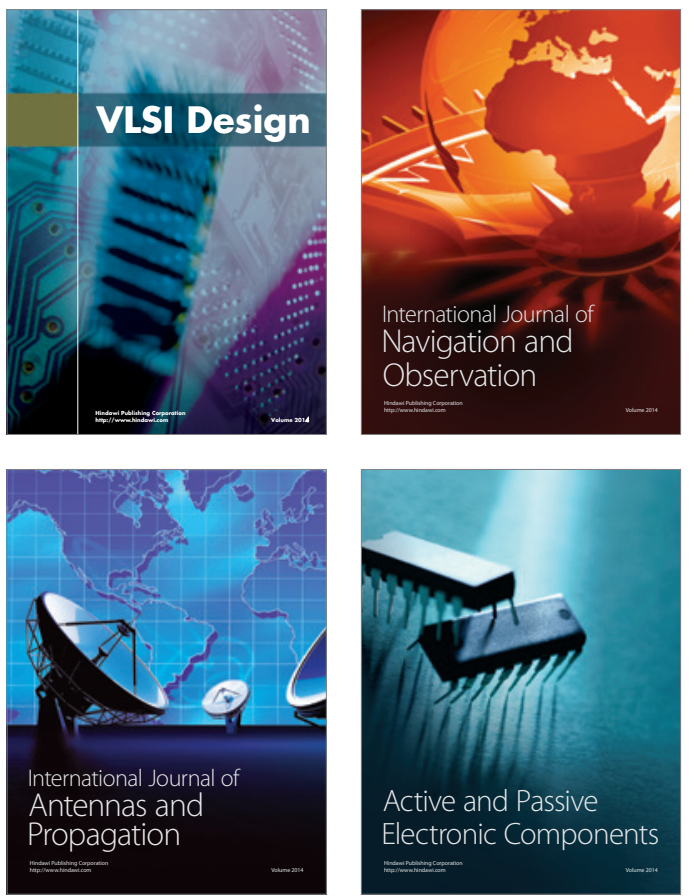
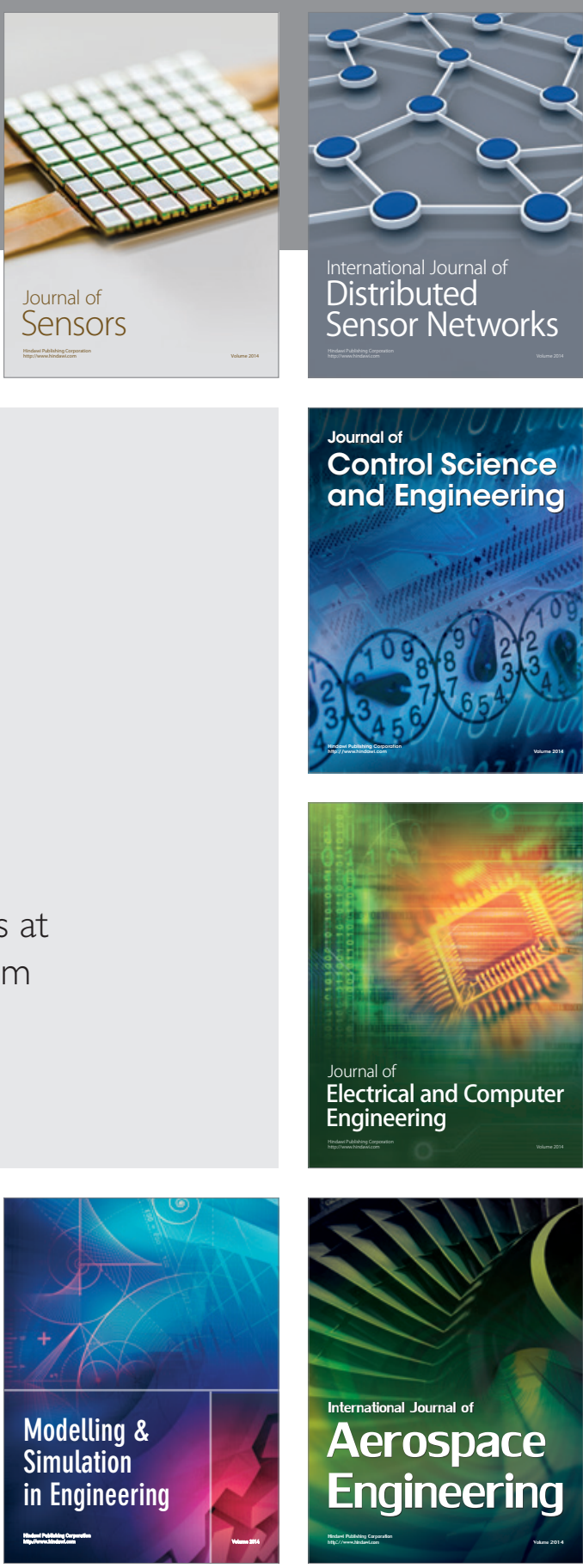

International Journal of

Distributed

Sensor Networks

$-$

Joumal of

Control Science

and Engineering
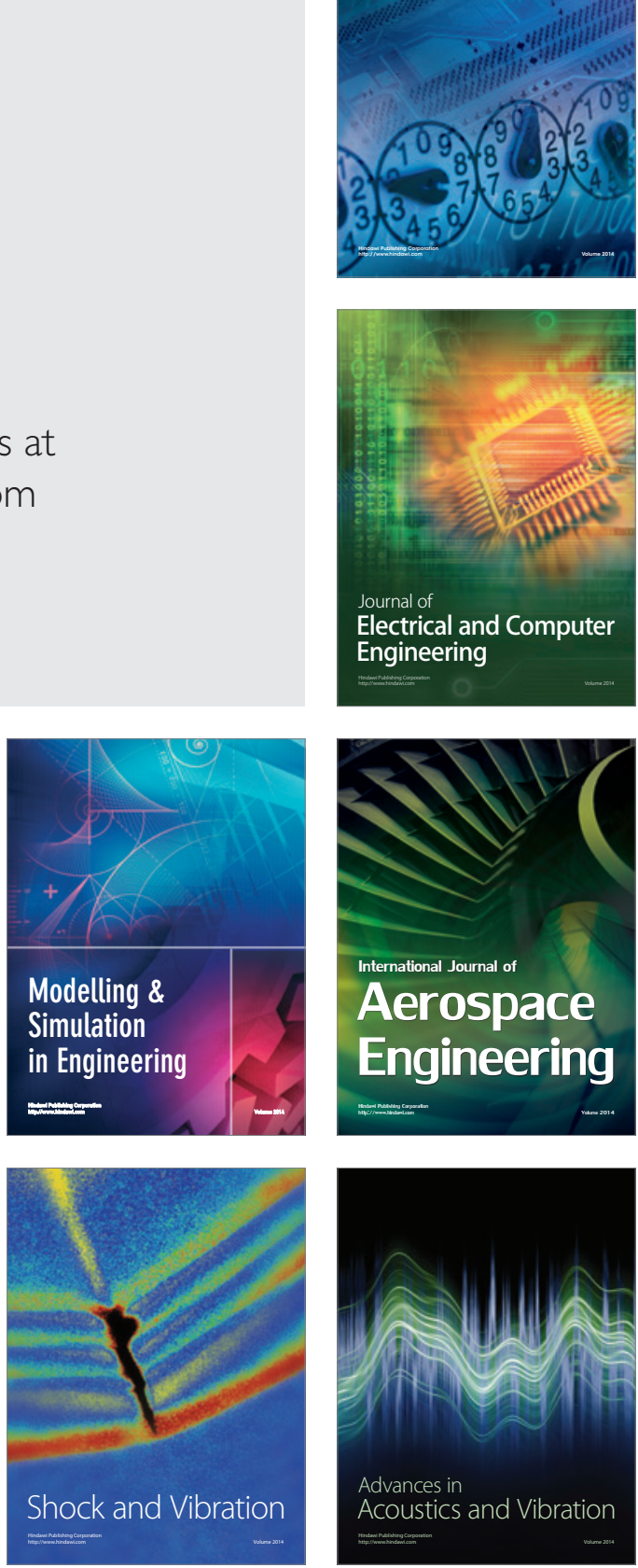